\title{
Measurement of $\boldsymbol{J} / \boldsymbol{\psi}, \boldsymbol{W}$ boson and $\boldsymbol{Z}$ boson in $\mathrm{Pb}-\mathrm{Pb}$ collisions at ATLAS *
}

\section{R. SANDSTRÖM FOR the ATLAS COLLABORATiOn}

\author{
Max-Planck-Institut für Physik, (Werner-Heisenberg-Institut), Föhringer Ring 6, \\ 80805 München, Germany
}

\begin{abstract}
A broad program of measurements using heavy ion collisions is underway in ATLAS, with the aim of studying the properties of QCD matter at high temperatures and densities. Heavy ion collisions produce a very large number of particles in the Inner Detector, but only muons traverse the calorimeter and leave tracks in the Muon Spectrometer, making muons essential tools for the study of heavy ion collisions. The centrality dependence of $J / \psi \rightarrow \mu^{+} \mu^{-}$yields allows the study of modification production of heavy quarkonium states. $W$ boson and $Z$ boson measurements are also possible using their decay leptons, and provide another handle on the initial state, and in particular the nuclear PDFs. This document describes measurements performed using $5-6.7 \mathrm{pb}^{-1}$ of $\mathrm{Pb}-\mathrm{Pb}$ collision data provided at a nucleon-nucleon center-of-mass energy of $2.76 \mathrm{GeV}$ by the Large Hadron Collider and collected by the ATLAS Detector during November and December 2010.
\end{abstract}

PACS numbers: 14.40.Lb, 14.70.Fm, 14.70.Hp, 25.75.Cj, 25.75.Dw

\section{Introduction}

In this document we present the first results by the ATLAS experiment [1] on the $J / \psi$ suppression as a function of collision centrality, the rate of $Z$ boson production measured in the di-muon channel [2] and the production of $W$ bosons in heavy ion collisions via the measurement of the inclusive muon $p_{\mathrm{T}}$ spectrum [3].

Muons are measured by combining independent measurements of the muon trajectories from the Inner Detector (ID) and the Muon Spectrometer (MS). A detailed description of these detectors and their performance in proton-proton collisions can be found in Refs. [1,4]. The ID volume is within the $2 \mathrm{~T}$ field of a superconducting solenoid, and measures the trajectories of

\footnotetext{
* Presented at Strangeness in Quark Matter 2011
} 
charged particles in the pseudorapidity region $|\eta|<2.5^{1}$. A charged particle typically traverses three layers of silicon pixel detectors, eight silicon strip sensors (SCT detector) arranged in four layers of double-sided modules, and a transition radiation tracker composed of straw tubes. The MS surrounds the ID and provides tracking for muons with $|\eta|<2.7$ and triggering in the range $|\eta|<2.4$. The muon momentum determination is based on three stations of precision drift chambers that measure the trajectory of each muon in a toroidal magnetic field produced by three air-core toroids. In order to reach the MS, muons have to cross the electromagnetic and hadronic calorimeters situated between the ID and MS , losing typically 3 to $5 \mathrm{GeV}$ of energy, depending on the muon pseudorapidity. The calorimeters efficiently absorb the copious hadrons produced in $\mathrm{Pb}-\mathrm{Pb}$ collision, and allow clean identification of muons.

The Level-1 minimum-bias trigger uses either the two sets of MinimumBias Trigger Scintillator (MBTS) counters, covering $2.1<|\eta|<3.9$ on each side of the experiment, or the two Zero-Degree Calorimeters (ZDC), each positioned at $140 \mathrm{~m}$ from the collision point, detecting neutrons and photons with $|\eta|>8.3$. The MBTS trigger was configured to require at least one hit above threshold from each side of the detector. The ZDC trigger threshold is set around the peak corresponding to single neutron ejected from $\mathrm{Pb}$ ions.

In the offline analysis, minimum-bias triggered events are required to have a reconstructed primary vertex, at least one hit in each set of MBTS counters, and a time difference between the sides of less than $3 \mathrm{~ns}$ to reject beam-halo and other beam-related background events. Measurements of the muon trajectories from both the ID and MS are combined, resulting in a relative momentum resolution ranging from about $2 \%$ at low momentum up to about $3 \%$ at $p_{\mathrm{T}} \sim 50 \mathrm{GeV}$. For this analysis, oppositely charged muons are selected with a minimum $p_{\mathrm{T}}$ of $3 \mathrm{GeV}$ each and within the region $|\eta|<2.5$.

The data sample used for the $W$ measurements consists of approximately $5 \mu \mathrm{b}^{-1}$ from the 2010 LHC heavy ion run, while the $J / \psi$ and $Z$ used $7.6 \mu \mathrm{b}^{-1}$. The centrality dependence of the muon reconstruction efficiency of muons from $W$-decays was evaluated using the hit multiplicities in the subdetectors for muon tracks with loosened selection criteria. In order to determine the $J / \psi \rightarrow \mu^{+} \mu^{-}$reconstruction efficiency, Monte Carlo (MC) samples have been produced superimposing $J / \psi$ and $Z$ events from

\footnotetext{
${ }^{1}$ In the right-handed ATLAS coordinate system, the pseudorapidity $\eta$ is defined as $\eta=-\ln [\tan (\theta / 2)]$, where the polar angle $\theta$ is measured with respect to the LHC beamline. The azimuthal angle $\phi$ is measured with respect to the $\mathrm{x}$-axis, which points towards the centre of the LHC ring. The z-axis is parallel to the anti-clockwise beam viewed from above. Transverse momentum and energy are defined as $p_{\mathrm{T}}=p \sin \theta$ and $E_{\mathrm{T}}=E \sin \theta$, respectively.
} 
PYTHIA [5] into simulated $\mathrm{Pb}-\mathrm{Pb}$ events generated with the HIJING [6] event generator. HIJING was run in a mode with effects from jet quenching turned off, since they have not been adjusted to agree with existing experimental data. Elliptic flow was imposed on the events subsequent to generation, with a magnitude and $p_{\mathrm{T}}$ dependence derived from RHIC data. The detector response to the complete PYTHIA+HIJING event is simulated [7] using GEANT4 [8].

$\mathrm{Pb}-\mathrm{Pb}$ collision centrality percentiles are defined from the total transverse energy, $\Sigma E_{\mathrm{T}}^{\mathrm{FCal}}$, measured in the forward calorimeter (FCal), which covers $3.2<|\eta|<4.9$. The centrality dependence of the muon detection efficiency is parameterized as a function of the total number of hits per unit of pseudorapidity detected in the first pixel layer. This is strongly correlated to $\Sigma E_{\mathrm{T}}^{\mathrm{FCal}}$, but gives a more direct measure of the ID occupancy. The full data sample is divided into four bins of collision centrality, 40-80\%, 20-40\%, $10-20 \%$, and $0-10 \%$. The most peripheral $20 \%$ of collisions are excluded from this analysis due to larger systematic uncertainties in estimating the number of binary nucleon-nucleon collisions in these events.

\section{Measurements}

$$
\text { 2.1. } J / \psi
$$

The oppositely-charged di-muon invariant mass spectra in the $J / \psi$ region after the selection are shown in Figure 1. The number of $J / \psi \rightarrow \mu^{+} \mu^{-}$ decays is then found by a simple counting technique. The signal mass window is defined by the range 2.95-3.25 GeV. The background is derived from two mass sidebands, $2.4-2.8 \mathrm{GeV}$ and $3.4-3.8 \mathrm{GeV}$, with a linear extrapolation. To determine the uncertainties related to the signal extraction, an alternative method based on a maximum likelihood fit with the mass resolution left as a free parameter is used as a cross check. Centrality-dependent efficiency corrections, derived from Monte Carlo events, are applied to the resulting signal yields. The number of $J / \psi$ decays after background subtraction, but before any other correction, are listed in Table 1 . With the chosen transverse momentum cuts on the decay muons, $80 \%$ of the reconstructed $J / \psi$ have $p_{\mathrm{T}}>6.5 \mathrm{GeV}$.

The measured $J / \psi$ yields at different centralities are corrected by the reconstruction efficiency $\epsilon_{c}$ for $J / \psi \rightarrow \mu^{+} \mu^{-}$, derived from MC and parameterized in each centrality bin, and the width of the centrality bin, $W_{c}$, which represents a well-defined fraction of the minimum bias events. The corrected yield of $J / \psi$ mesons is given by:

$$
N_{c}^{\text {corr }}\left(J / \psi \rightarrow \mu^{+} \mu^{-}\right)=\frac{N^{\text {meas }}\left(J / \psi \rightarrow \mu^{+} \mu^{-}\right)_{c}}{\epsilon(J / \psi)_{c} \cdot W_{c}} .
$$




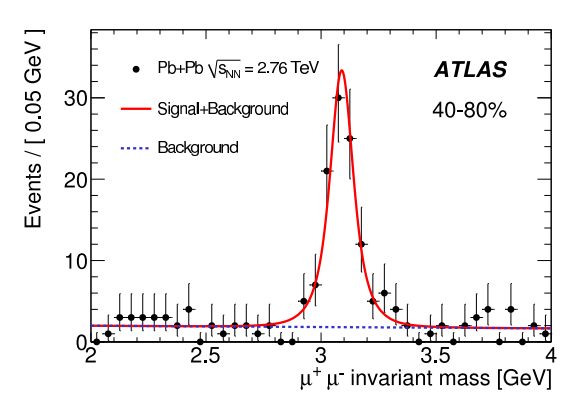

(a) $(40-80 \%)$

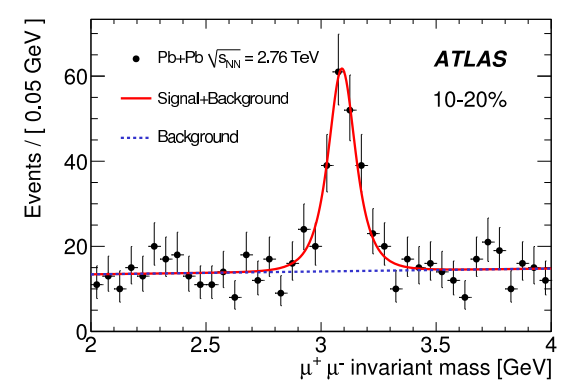

(c) $(10-20 \%)$

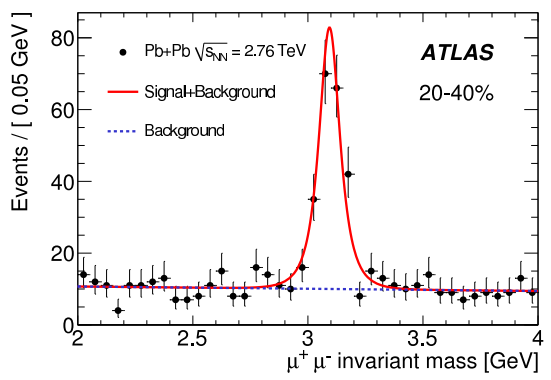

(b) $(20-40 \%)$

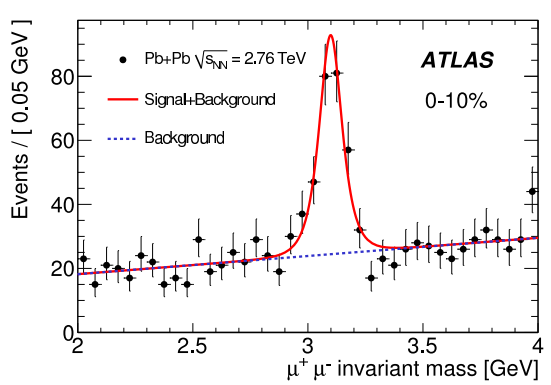

(d) $(0-10 \%)$

Fig. 1. Oppositely-charged di-muon invariant mass spectra in the four considered centrality bins from most peripheral (40-80\%) to most central (0-10\%). [2] The $J / \psi$ yields in each centrality bin are obtained using a sideband technique. The fits shown here are used as a cross check.

The normalized yield is defined by scaling the corrected yield by the ratio $R_{\text {coll }}$ of the mean number of binary collisions $N_{\text {coll }, c}$ in each centrality bin to that for the most peripheral (40-80\%) bin: $R_{C P}=N_{c}^{\text {corr }} /\left(N_{40-80 \%}^{\text {corr }} R_{\text {coll }, C P}\right)$. The resulting $R_{C P}$ is shown in Fig. 2(a). A strong suppression as a function of collision centrality is observed. The $p$-value for obtaining this result in the case of no suppression is $0.11 \%$.

\subsection{Z-boson}

$Z$-boson candidates are selected by requiring a pair of oppositely charged muons with $p_{\mathrm{T}}>20 \mathrm{GeV}$ and $|\eta|<2.5$ [9]. An additional cosmic ray rejection cut on the sum of the pseudorapidities of the two muons, $\mid \eta_{1}+$ $\eta_{2} \mid>0.01$, is also applied. No cosmic candidates were identified. The invariant mass distribution of the selected pairs is shown in the left panel of Figure 2(b). With this selection, $38 Z$ candidates are retained in the 


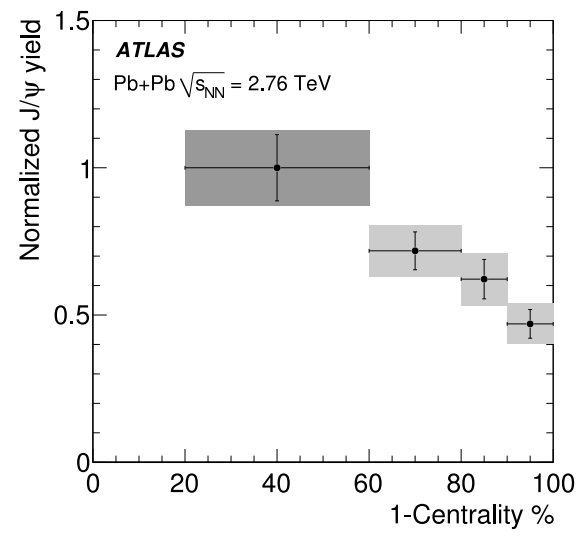

(a) $J / \psi \rightarrow \mu \mu$

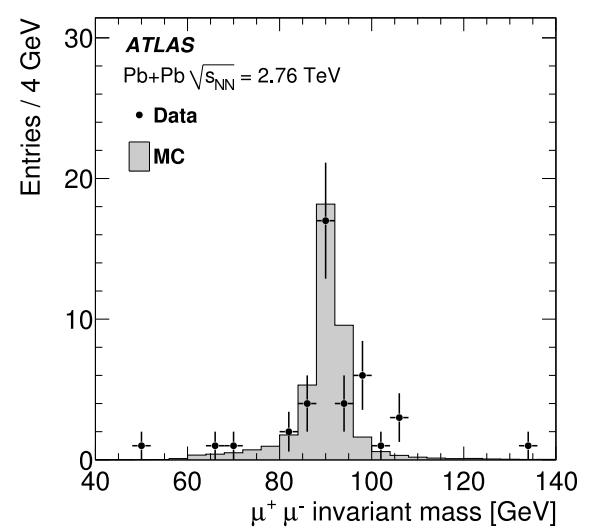

(b) $Z \rightarrow \mu \mu$

Fig. 2. (a) Value of $R_{C P}$ for $J / \psi$ events as a function of centrality [2]. The 40$80 \%$ bin is used to set the scale for all bins, but the uncertainties in this bin are not propagated into the more central ones. The statistical errors are shown as vertical bars while the filled boxes also include the combined systematic errors. (b) Dimuon invariant mass using $6.7 \mu \mathrm{b}^{-1}[2]$. A resonance is found at an invariant mass corresponding to the $Z$ boson mass.

signal mass window of 66 to $116 \mathrm{GeV}$. The background after this selection is expected to be below $2 \%$, and is not corrected for in the result. The number of $Z$ events in each centrality bin is given in Table 1 .

\section{3. $W$-boson}

In heavy ion collisions, the missing- $E_{\mathrm{T}}$ resolution and the muon isolation requirements are strongly dependent on the collision centrality and are therefore not reliable in $\mathrm{Pb}-\mathrm{Pb}$ collisions. We obtain $W$ production yields by simultaneous fits of the muon $p_{\mathrm{T}}$ spectra, shown in Fig 3(a), to two input shapes. The first shape describes the muon $p_{\mathrm{T}}$ distribution from $W^{ \pm} \rightarrow \mu^{ \pm} \nu$, and is obtained from simulations of $p p \rightarrow W^{ \pm}$at $2.76 \mathrm{TeV}$. The second shape describe the decay background of $c \bar{c} \rightarrow \mu+X$ and $b \bar{b} \rightarrow \mu+X$ and is parameterized from PYTHIA samples. The fit is done using the unbinned maximum log-likelihood method, and the amplitude of the $W$-decay curve gives the number of $W \rightarrow \mu \nu$ events.

\subsection{1. $W$ yield as function of centrality}

Yields for the four centrality bins are obtained from fits to the corresponding muon $p_{\mathrm{T}}$ spectrum. The results are shown in Table 1. To compare 


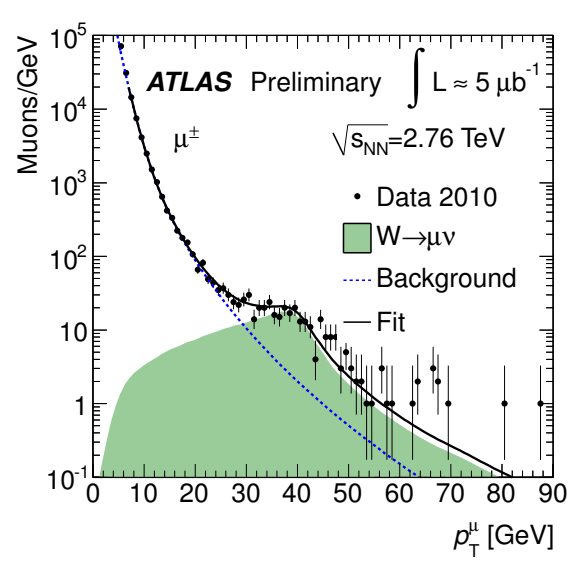

(a) $p_{\mathrm{T}}$

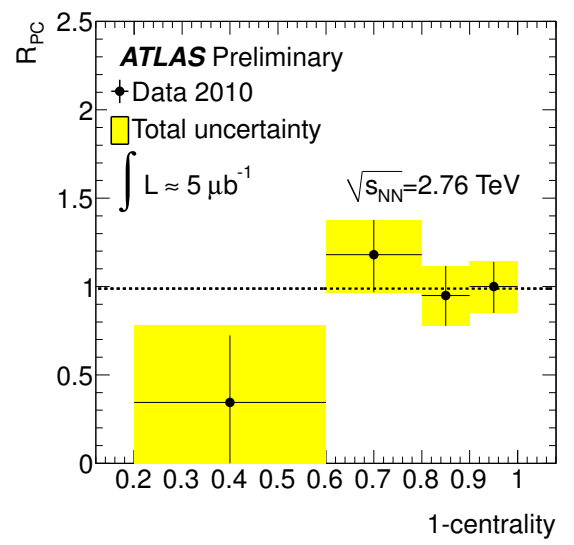

(b) $R_{P C}$

Fig. 3. W measurements in $\mathrm{Pb}+\mathrm{Pb}$ collisions. (a) Uncorrected inclusive muon $p_{\mathrm{T}}$ spectrum. [3]. The spectrum is fitted (solid line) with two components: signal $W \rightarrow \mu \nu$ (shaded area) simulated with PYTHIA [5] in pp collisions at $\sqrt{s_{N N}}=$ $2.76 \mathrm{TeV}$, and a background parametrization (dashed line) obtained from studies of $c \bar{c} \rightarrow \mu+X$ and $b \bar{b} \rightarrow \mu+X$ decays in $p p$ simulations. (b) Value of $R_{P C}$ for $W$ events as a function of centrality [3]. Yields for each centrality bin are normalized to the counts of the most central bin. The dashed line is the result of a fit to a constant value to test the hypothesis for the absence of suppression.

Table 1. Uncorrected measured numbers of $W, J / \psi$ and $Z$ events per centrality bin as obtained from the fit method [3]. The total number of $W$ bosons is from a fit to the inclusive muon $p_{\mathrm{T}}$ spectrum. Uncertainties come mainly from the fit procedure and statistics. The number of $J / \psi$ events is extracted by a counting method [2], while the $Z$ events is a simple event count. Ratios of the mean number of binary collisions for the most peripheral events to other centralities $i, R_{\text {coll, CP }}^{W}=<N_{\text {coll }}^{i}>$ $\left./<N_{\text {coll }}^{P}\right\rangle$, and corresponding uncertainties are also indicated. This was used for the $W$ analysis. $R_{\text {coll, } P C}^{J / \psi, Z}=<N_{\text {coll }}^{C}>/<N_{\text {coll }}^{i}>$ is the same ratio normalized to the $10 \%$ most central events, which was used for the $J / \psi$ and $Z$ analyses.

\begin{tabular}{lcccc}
\hline Selection & $0-10 \%$ & $10-20 \%$ & $20-40 \%$ & $40-80 \%$ \\
\hline$N_{J / \psi}$ & $190 \pm 20$ & $152 \pm 16$ & $180 \pm 16$ & $91 \pm 10$ \\
$N_{Z}$ & 19 & 5 & 10 & 4 \\
$N_{W}$ & $165_{-25}^{+23}$ & $97_{-18}^{+16}$ & $118_{-24}^{+17}$ & $12_{-12}^{+13}$ \\
$R_{\text {coll, } C P}^{W}$ & $19.3^{W} \pm 1.4$ & $11.9 \pm 0.7$ & $5.7 \pm 0.3$ & 1.0 \\
$R_{\text {coll }, P C}^{J / P}$ & 1.0 & $1.6 \pm 0.0$ & $3.4 \pm 0.1$ & $19.3 \pm 1.4$ \\
\hline
\end{tabular}


the relative number of events produced at each centrality bin we normalize the observed number of $W$ in Table 1 to the counts from the most central bin, using the ratios of mean numbers of binary collisions. In Fig. 3 (b), we show the number of $W$ events normalized to central $W$ events, $R_{P C}$, for all of centrality bins. Fitting the data to a constant value returns $<R_{P C}>=0.99 \pm 0.10$ with a $\chi^{2}=3.02$ for 3 degrees of freedom. Therefore this observation is an indication that $W$ bosons are indeed produced at the initial phase of the collisions and do not interact with the medium.

\subsection{2. $W^{+} / W^{-}$and $W / Z$ ratios}

The larger number of $d$ valence quarks over $u$ valence quarks in $\mathrm{Pb}$ ions favors the production of $W^{-}$bosons over $W^{+}$in $\mathrm{Pb}-\mathrm{Pb}$ collisions at the LHC. The expected ratio according to theory is $R_{W^{+}} / W^{-}=0.90 \pm 0.05$ without the nuclear modification of the PDF [10]. The ratio obtained from our data is

$$
R_{W^{+} / W^{-}}=\frac{198_{-26}^{+25}}{204_{-31}^{+27}}=0.97_{-0.19}^{+0.18}
$$

for the ATLAS pseudorapidity acceptance. Higher statistics will permit a better measurement and could provide a good target for model testing.

The measurement of the ratio of $W \rightarrow \mu \nu$ to $Z \rightarrow \mu^{+} \mu^{-}$yields is an important test of the Standard Model. For the $W / Z$ ratio, we extract the number of $Z$ bosons applying the same event selection criteria used to reconstruct muons from $W$ decays. The ratio is found to be $R_{W / Z}^{f i t}=$ $10.5_{-2.4}^{+2.3}$. Systematic uncertainties due to acceptance and efficiency effects are included in the calculation, but the uncertainty is dominated by the small number of $Z$-bosons in the data set. Calculations from Ref. [10] of $W$ and $Z$ boson production cross sections in $\mathrm{Pb}-\mathrm{Pb}$ collisions, with or without nuclear modifications of the $\mathrm{PDF}$, give a ratio $R_{W / Z}=11.5 \pm 0.7$.

\section{Conclusion}

We conclude from the absence of suppression of the number of $W \rightarrow$ $\mu \nu$ events as function of collision centrality that neither the $W$ bosons, nor the decay muons, interact with the medium. The data also show no evidence of $Z$ suppression, albeit with very large statistical uncertainties. The $J / \psi \rightarrow \mu^{+} \mu^{-}$is however rather strongly suppressed, thus confirming the results of previous experiments [11, 12]. The observed ratios $R_{W^{+} / W^{-}}$ and $R_{W / Z}$, agree with Standard Model predictions [10,13]. This is the first time that these measurements have been done in nucleus-nucleus collisions. With more data, these measurements will provide sensitive tests of $W$ and 
$Z$ boson production models, in particular possible nuclear modifications to the parton distribution functions.

\section{REFERENCES}

[1] ATLAS Collaboration. The ATLAS Experiment at the CERN Large Hadron Collider. JINST, 3:S08003, 2008.

[2] ATLAS Collaboration. Measurement of the centrality dependence of $\mathrm{J} / \psi$ yields and observation of $\mathrm{Z}$ production in lead-lead collisions with the ATLAS detector at the LHC. Phys.Lett., B697:294-312, 2011.

[3] ATLAS Collaboration. Measurements of $\mathrm{W}$ Boson Yields in $\mathrm{Pb}+\mathrm{Pb}$ at 2.76 $\mathrm{TeV} /$ nucleon via single muons with the ATLAS detector. (ATLAS-CONF2011-078), May 2011. http://cdsweb.cern.ch/record/1353227.

[4] ATLAS Collaboration. Expected performance of the atlas experiment: detector, trigger and physics. CERN-OPEN-2008-020, 2009.

[5] Torbjorn Sjostrand, Stephen Mrenna, and Peter Z. Skands. PYTHIA 6.4 Physics and Manual. JHEP, 0605:026, 2006.

[6] Xin-Nian Wang and Miklos Gyulassy. HIJING: A Monte Carlo model for multiple jet production in p p, p A and A A collisions. Phys.Rev., D44:35013516, 1991.

[7] ATLAS Collaboration. The ATLAS Simulation Infrastructure. Eur.Phys.J., C70:823-874, 2010.

[8] S. Agostinelli et al. GEANT4: A Simulation toolkit. Nucl.Instrum.Meth., A506:250-303, 2003.

[9] ATLAS Collaboration. Measurement of the $W \rightarrow \ell \nu$ and $Z \rightarrow \ell \ell$ production cross-sections in proton-proton collisions at $\sqrt{s}=7 \mathrm{TeV}$ with the ATLAS detector. arXiv:1010.2130 [hep-ex], 2010. Accepted by JHEP.

[10] Hannu Paukkunen and Carlos A. Salgado. Constraints for the nuclear parton distributions from $\mathrm{Z}$ and $\mathrm{W}$ production at the LHC. JHEP, 1103:071, 2011.

[11] B. Alessandro et al. A New measurement of $\mathrm{J} / \mathrm{psi}$ suppression in $\mathrm{Pb}-\mathrm{Pb}$ collisions at 158-GeV per nucleon. Eur.Phys.J., C39:335-345, 2005.

[12] A. Adare et al. J/psi Production vs Centrality, Transverse Momentum, and Rapidity in $\mathrm{Au}+\mathrm{Au}$ Collisions at $\mathrm{s}(\mathrm{NN})^{* *}(1 / 2)=200-\mathrm{GeV}$. Phys.Rev.Lett., 98:232301, 2007.

[13] R. Vogt. Shadowing effects on vector boson production. Phys.Rev., C64:044901, 2001. 